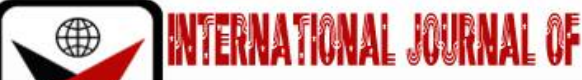

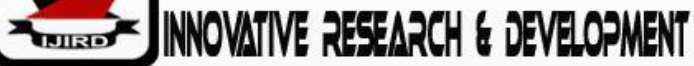

ISSN 2278 - 0211 (Online)

\section{Sedimentological and Physicochemical Properties of Sediments from .Oblaruku and Environ, Southwest Nigeria}

\author{
Odumoso, Solomon Emeke \\ Assistant Lecturer, Department of Earth Sciences, \\ Federal University of Petroleum Resources, Effurun, Delta State, Nigeria
}

\begin{abstract}
:
Sedimentological and some physicochemical analyses were carried out on soil samples within Obiaruku and environs in order to establish baseline conditions for the purpose of basic development projects. The results show that the sediments were of medium grade, poorly to moderately sorted and finely to strongly coarse skewed with a leptokurtic to platykurtic kurtosis. Effective cation exchange capacity and carbon content was generally low ranging between 8.68-17.88ppm and 0. 70-3.05\% respectively. Phosphorus contents ranged from 0.40-1.04\% and 0.20-1.20\% for surface and subsurface soils respectively. Phosphorus availability in the soil was observed to be influenced by the soil pH, potassium, and Effective cation exchange capacity. Similarly, Nitrate content ranged from $0.03-0.14 \%$ and $0.02-$ $0.09 \%$ for surface and subsurface soils respectively. The availability of nitrogen in the soil was influenced by the presence of potassium only among the parameters tested. Phosphate and nitrate content in the soils also decreased with depth. Changes in soil chemistry were related to natural variations in grain size, organic matter content and geologic substrate. Within the subsurface, the study area shows a fairly low nutrient concentration as against $0.02 \%$ in natural soils to $0.15 \%$ in cultivated soils for nitrogen, and less than $1.5 \%$ in soils for available phosphorus.
\end{abstract}

Keywords: Phosphorus, nitrate, soil, Nigeria

\section{Introduction}

Sediments act as a store house of huge information ci tae varied activities around us. 'While nitrogen in soils occurs in several forms as organic compounds, ammonium ions, nitrate, nitrite, nitrous acid and nitrogen gas (Ikomi, 2000). The largest concentration of nitrogen in surface soils is in the organic form. Beside nitrogen being added to the soil through biological nitrogen fixation in a slightly acidic to neutral soil, most plants take up the nitrate form of nitrogen.

Phosphorus on the other hand is one of the major essential elements required by crops for normal growth. Among the macronutrients with the exception of nitrogen, no other element is as critical in agriculture as is phosphorus. Phosphorus and nitrogen primarily in the oxidized forms of phosphate ( $\mathrm{P}^{3} 4^{3}$ ) and nitrate (NOC) can be used as indicators both for population (municipal waste) and agriculture impact on the environment. Based on studies carried out by Adepeju et al (1970), the nitrogen and phosphorus levels seem to change very little, due to surface processes that subsequently lead to the contamination of the surface and the subsurface water bodies. The organic phosphorus normally accounts for $20-50 \%$ of the top phosphorus and may be affected significantly by the surface processes. The removal of phosphorus from soils into local waters tends to be associated most with the removal of adsorbed phosphorus on soil sediments by erosion than by the leaching of phosphorus in solution to the underground water table, which may contaminate it. This research therefore seeks to study the sediment logic and selected physicochemical characteristics on soil samples within Obiaruku and environs in order to establish baseline conditions for the purpose of basic development projects

\section{Study Area}

The study area is located in the Niger Delta Basin of Nigeria between latitude $50^{0} 31 \mathrm{WI}$ and $\left.50^{\circ} 45\right\} \mathrm{N}$ and longitude $60^{\circ} 2^{1} \mathrm{E}$ and $60^{0} 10^{1} \mathrm{E}$. It is within the subequatorial region characterized by alternate wet and dry seasons. The area is generally low lying, sloping slightly seaward and with elevation of $20 \mathrm{~m}$ above sea level and drained by the River Ethiope and its tributaries. The area comprises of several medium sized towns arid vast farmlands located on the top of the coastalplailk (Benin Formation). Soil type varies from white, dark brown to light red (Kaizer and Adaikpoh 2006; Kaizer 2007a; Kaizer 2007b). Like many towns in Nigeria, the study area lacks planned network drainage. Nearly all the road networks (both major and minor).

Lack roadside drains. As a result, surface run-offs contaminated with municipal waste drain directly into the associated farmlands and River Ethiope. Generated waste types include among others animal waste, trash, dump form old dumpsites. Major communities and population sizes within the study area include Obiaruku (115,686 people), Umutu (9,5448 people), Umuaja (7,563 people) and Abavo (12,346 people) (FRN Census 1991). With rapid population growth, 
the major economic activity in the area remains crop and animal agriculture and commodity trading. The agricultural practice found in the area is mainly subsistence and the dominant crops cultivated include yam, cassava and maize. Most of the lands in the area is forested and serve for conservation of wild life and natural resources. Umuaja, the source of River Ethiope serves as recreational center.

Generally, about fifty percent (50\%) of the water in the study area is used for domestic and commercial purposes. Only above five percent (5\%) of the water in the area is used for industrial purposes for processing of materials. Major industries include the Michelin rubber industry at Utagba-Uno, Nigerian Hardwood Company at Obiaruku State University at Abraka, Rubber Research Estate at Urhonigbe and many small scale oil palm processing Factories. Groundwater accounts for about $14 \%$ of total water used. The Ethiope and Warri rivers and private domestic wells are the dependable sources of water in areas not served by public water supplies.

\section{Methodology}

Soil samples were collected randomly from the surface and profile pits dug within the study area. The samples were collected in polythene bags with the aid of a cylindrical stainless steel iron hand corer at depth of $0-30 \mathrm{~cm}$ from the surface and $3 \mathrm{ft}$ interval within the profile pits. The samples were then labeled with the date of collection, sample identification number and location recorded appropriately. Samples analyzed for nitrate content were preserved in sulphuric acid with a pH of less than two and stored in the refrigerator at a temperature of $4^{\circ} \mathrm{C}$. On the other hand, samples analyzed for phosphate content were similarly stored in the refrigerator at a temperature of $4^{\circ} \mathrm{c}$, without adding preservatives. The soil samples were air dried, sieved with a $2 \mathrm{~mm}$ mesh size and then subjected to physical and chemical analyses. Particle size distribution was determined by the hydrometer method (Bouyoucos, 1962) using 5\% sodium hexamethaphosphate as a soil-dispersing agent. Soil $\mathrm{pH}$ was determined in soil-water suspension at 1:2 soil solution ratio using glass electrode pH meter. Total and available nitrogen was determined by Bray No. I method (Barry and Kurtz, 1955). Total exchangeable cation and effective exchange capacity where determined by extracting the soil within INH40AC (Scollenberger and Simon, 1945). Sodium, calcium, and potassium were determined by Atomic absorption spectrometer (AAS). Summation of exchangeable acidity gave the Effective cation exchange capacity value.

\section{Results and Discussion}

The result of the analysis carried out on the soil samples are presented in Tables 1-3. Size distribution varied considerably among locations. Soil texture varied from poorly sorted through moderately well sorted. A fining upward sequence was observed in the area. Soil pH varied from moderately to weakly acidic $4.31-5.83$ for surface soils (mean 5.15), while subsurface soils ranged from 4.75-5.54 (means 5.19). Generally, pH increased with depth. Carbon content of the surface soils ranged from $0.70 \%-3.05 \%$ (mean $1.48 \%$ ) while subsurface soils ranged from $0.06-2.85 \%$ (mean $0.71 \%$ ). The carbon content in the surface soils was higher than the subsurface soils. Effective cation exchange capacity (ECEC) of the surface soils ranged from 8.63-18.73ppm (mean 14.32ppm) while that of the subsurface soils varied from 3.1012.32ppm (mean 7.05ppm). ECEC of the surface soils were higher than that of the subsurface soils. Phosphorus content ranged from $0.04-1.04 \%$ (mean $0.71 \%$ ), and $0.20-1.20 \%$ (mean $0.57 \%$ ) in the surface and subsurface soils respectively. Nitrogen content of the surface soils varied form $0.03-0.14$ (mean $0.08 \%$ ) and $0.02-0.09 \%$ (mean $0.04 \%$ ) in the subsurface soil. Both the available phosphorus and nitrogen in the oxidized forms of phosphates and nitrates can be used as indicators both of population and agricultural impact on the environment. The variations of phosphate and nitrate content in soils within the study area were characteristics of multiple human impacts.

Sedimentologic correlation of the profiles displayed a uniform structure and chemical characteristics from the top to bottom. Soil type varied slightly with grain size distribution mainly between medium to coarse grains. Although few local clay deposits occurred in the area, they were insignificant as they were only found as local deposit along the River Ethiope channel. Generally, the chemical characteristics of the sediments were greatly influenced by the soil texture and land use pattern within the area. Also, the degree of porosity and permeability exhibited by the soil encouraged the leaching of the upper soil nutrients into depths with high rainfall. Available phosphorus in the surface soils ranged from $0.03-0.14 \%$ and $0.02-0.09 \%$ for the surface and subsurface soils respectively. $\mathrm{pH}$ appeared to be the major factor that determined the presence of available phosphorus while potassium solely affected the availability of nitrogen content in the soils.

\section{Conclusion}

Changes in soil chemistry were related to natural variations in grain size, organic matter content and geologic substrate. Within the subsurface, the study area shows a fairly low nutrient concentration as against $0.02 \%$ in natural soils to $0.15 \%$ in cultivated soils for nitrogen (Kent, 1998), and less than $1.5 \%$ in soils for available phosphorus (Agboola and Corey, 1976; Kent 2001). This result therefore presents the area as not significantly impacted. 


\begin{tabular}{|c|c|c|c|c|c|c|c|c|c|c|}
\hline Parameters & $\begin{array}{l}\text { ABR } \\
\text { AKA } \\
\text { S3B } \\
\end{array}$ & $\begin{array}{c}\text { OBIAR } \\
\text { UKU } \\
\text { SIB } \\
\end{array}$ & $\begin{array}{c}\text { OBIAR } \\
\text { UKU } \\
\text { S4A } \\
\end{array}$ & $\begin{array}{l}\text { ABR } \\
\text { AKA } \\
\text { S3A } \\
\end{array}$ & $\begin{array}{c}\text { OBIAR } \\
\text { UKU } \\
\text { S1A }\end{array}$ & $\begin{array}{l}\text { ABR } \\
\text { AKA } \\
\text { SAB } \\
\end{array}$ & $\begin{array}{c}\text { ABRA } \\
\text { KA } \\
\text { S5B } \\
\end{array}$ & $\begin{array}{c}\text { OBIAR } \\
\text { UKU } \\
\text { S2B } \\
\end{array}$ & $\begin{array}{c}\text { OBIAR } \\
\text { UKU } \\
\text { S4B } \\
\end{array}$ & UMUAJA \\
\hline PH & 5.60 & 5.338 & 5.01 & 5.01 & 5.17 & & 5.13 & 4.93 & 5.13 & 5.46 \\
\hline Carbon $(0 / 0)$ & 1.51 & I. 43 & 1.32 & 1.11 & 1.45 & & 0.70 & 1.30 & 1.43 & 1.48 \\
\hline Nitrogen (\%) & 0.12 & 0.11 & 0.06 & 0.07 & 0.06 & 0.04 & 0.04 & 0.08 & 0.09 & 0.14 \\
\hline $\begin{array}{c}\text { Available } \\
\text { Phosphorous } \\
(\%)\end{array}$ & 0.50 & 0.59 & 0.98 & 0.90 & 1.05 & 0.60 & 0.60 & 0.50 & 0.40 & 1.04 \\
\hline Sodium (ppm) & 0.24 & 0.24 & 0.25 & 0.30 & 0.50 & 0.17 & 0.17 & 0.27 & 0.23 & 0.23 \\
\hline $\begin{array}{c}\text { Conductivity } \\
\left(\mu \mathrm{cm}^{-1}\right)\end{array}$ & 53.40 & 66.50 & 41.22 & 33.46 & 59.07 & 43.18 & 43.18 & 38.44 & 36.05 & 38.07 \\
\hline $\begin{array}{c}\text { Potassium } \\
\text { (ppm) }\end{array}$ & 0.09 & 0.17 & 0.12 & 0.20 & 0.05 & 0.05 & 0.05 & 0.7 & 0.15 & 0.15 \\
\hline Sodium (ppm) & 3.10 & 3.20 & 6.10 & 6.50 & 5.00 & 4.30 & 3.10 & 3.40 & 5.10 & 4.10 \\
\hline $\begin{array}{c}\text { Magnesium } \\
(\mathrm{ppm})\end{array}$ & 1.06 & 1.15 & 0.47 & 0.51 & -31 & 0.29 & 0.73 & 1.16 & 1.12 & 0.75 \\
\hline ECEC (ppm) & 12.35 & 16.60 & 17.88 & 10.92 & 15.80 & 8.68 & 13.83 & 17.29 & 15.72 & 15.15 \\
\hline Base Status (\%) & 38.0 & 62.55 & 50.15 & 59.80 & 70.15 & 73.80 & 59.01 & 81.45 & 63.44 & 62.15 \\
\hline $\begin{array}{c}\text { Ammonia } \\
(\mathrm{ppm})\end{array}$ & 15.21 & 1420 & 20.40 & 22.50 & 18.50 & 2200 & 14.50 & 16.30 & 17.80 & 16.75 \\
\hline Nitrate (ppm) & 0.08 & 0.30 & 0.16 & 0.04 & 0.03 & 0.05 & 1.00 & 0.07 & 0.40 & 0.30 \\
\hline
\end{tabular}

Table 1: Chemical Analysis of Surface Soil Samples (PPM)

\begin{tabular}{|c|c|c|c|c|c|c|c|c|c|c|}
\hline Parameters & $\begin{array}{c}\text { ABRAK } \\
\text { A S3B }\end{array}$ & $\begin{array}{c}\text { OBLARU } \\
\text { KU SIB }\end{array}$ & $\begin{array}{c}\text { OBIARU } \\
\text { KU S4A }\end{array}$ & $\begin{array}{c}\text { ABRAK } \\
\text { A S3 }\end{array}$ & $\begin{array}{c}\text { OBIARU } \\
\text { KU } \\
\text { SIA }\end{array}$ & $\begin{array}{c}\text { ABRAK } \\
\text { A } \\
\text { S4B }\end{array}$ & $\begin{array}{c}\text { ABRAK } \\
\text { A S5B }\end{array}$ & $\begin{array}{c}\text { OBIARU } \\
\text { KU 32B }\end{array}$ & $\begin{array}{c}\text { OBLARU } \\
\text { KU S4B }\end{array}$ & UMUAJA \\
\hline $\mathrm{pH}$ & 5.23 & 5.14 & 4.68 & 5.09 & 5.05 & 5.15 & 4.31 & 5.83 & 5.17 & 5.39 \\
\hline $\mathrm{C} \%$ & 1.56 & .26 & 2.35 & 0.42 & $\mathrm{I} .33$ & 0.83 & 2.71 & 1.24 & 1.37 & 1.31 \\
\hline $\mathrm{N} \%$ & 0.07 & 0.09 & 0.05 & 0.07 & 0.08 & 0.12 & 0.12 & 0.14 & 0.06 & 0.04 \\
\hline $\mathrm{AP} \%$ & 0.55 & 0.62 & 0.03 & 0.85 & 1.00 & 0.60 & 0.57 & 0.52 & 0.73 & 0.91 \\
\hline $\mathrm{N}$ & 0.23 & 0.27 & 0.12 & 0.30 & 0.21 & 0.24 & 0.23 & 0.23 & 0.29 & 0.24 \\
\hline $\begin{array}{c}\text { Conductivity } \\
\left(\mu \mathrm{cm}^{-1}\right)\end{array}$ & 46.19 & 36.04 & 36.24 & 36.08 & 50.63 & 34.40 & 21.32 & 33.73 & 45.21 & 42.72 \\
\hline $\mathrm{K}$ & 0.02 & 0.15 & 0.13 & 0.20 & 0.13 & 0.10 & 0.09 & 0.12 & 0.13 & 0.19 \\
\hline $\mathrm{Ca}$ & 3.50 & 4.10 & 2.30 & 6.20 & 5.10 & 7.40 & 4.00 & 5.00 & 7.60 & 6.40 \\
\hline $\mathrm{Mg}$ & 0.34 & 0.61 & 0.33 & 1.21 & 0.37 & 0.82 & 0.57 & .0 .44 & $\mathrm{I} .44$ & 0.83 \\
\hline $\mathrm{ECEC}$ & 15.70 & 18.73 & 12.13 & 14.40 & 174.50 & 15.45 & 12.01 & 10.11 & 14.35 & 16.00 \\
\hline Base Status & 61.21 & 68.73 & 70.90 & 75.04 & 40.82 & 69.13 & 71.07 & 70.98 & 89.40 & 69.43 \\
\hline $\mathrm{NH}_{4}$ & 19.20 & 12.80 & 19.40 & 3.10 & 13.33 & 15.50 & 15.60 & 19.50 & 16.50 & 28.60 \\
\hline $\mathrm{NO}_{3}$ & 0.40 & 0.18 & 0.17 & 0.15 & 0.16 & 0.12 & 0.81 & 1.20 & 0.31 & 0.46 \\
\hline
\end{tabular}

Table 2: Chemical Analysis of Surface Soil Samples (PPM)

\begin{tabular}{|c|c|c|c|c|c|c|c|c|c|c|c|c|c|c|}
\hline 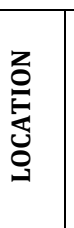 & 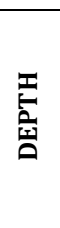 & 줄 & ذ̊ & ì & $\begin{array}{ll}\circ \\
\dot{0}\end{array}$ & z & 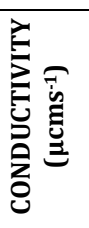 & 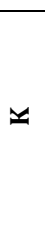 & & $\sum^{\infty}$ & 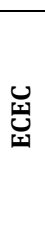 & 密 & $\frac{\mathbb{Z}^{*}}{\mathrm{z}}$ & $\stackrel{\infty}{2}$ \\
\hline \multirow{4}{*}{ 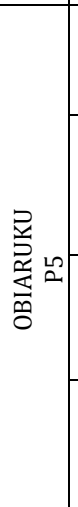 } & $\stackrel{\vec{m}}{m}$ & जे & 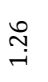 & $\stackrel{\text { : }}{0}$ & $\begin{array}{l}\infty_{1} \\
o^{\prime}\end{array}$ & $\begin{array}{l}\text { qu } \\
\text { : }\end{array}$ & 宛 & $\begin{array}{l}\hat{0} \\
0\end{array}$ & 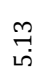 & 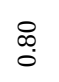 & $\stackrel{\substack{\infty \\
\infty}}{\infty}$ & $\begin{array}{l}q_{1}^{\prime} \\
\infty^{\prime}\end{array}$ & 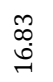 & $\stackrel{\circ}{0}$ \\
\hline & $\stackrel{\Delta}{\infty}$ & $\stackrel{m}{\dot{n}}$ & : & $\stackrel{\text { ¿े }}{0}$ & 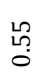 & $\vec{m}$ & 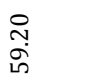 & $\begin{array}{l}\infty \\
0 \\
0 \\
0\end{array}$ & 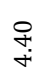 & 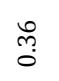 & 号 & $\underset{\substack { 0 \\
\begin{subarray}{c}{\infty \\
\infty{ 0 \\
\begin{subarray} { c } { \infty \\
\infty } }\end{subarray}}{\infty}$ & $\begin{array}{l}\stackrel{+}{+} \\
\stackrel{+}{*}\end{array}$ & $\stackrel{\substack{\sim \\
0}}{0}$ \\
\hline & $\underset{\sim}{\stackrel{\vec{N}}{N}}$ & تี & $\stackrel{\tilde{N}}{0}$ & $\stackrel{m}{0} \stackrel{0}{0}$ & ્ָ & $\stackrel{\text { İ }}{\text { Oे }}$ & 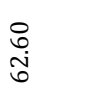 & ¿̊. & $\underset{+}{\stackrel{-}{~}}$ & $\stackrel{\mathscr{m}}{\stackrel{0}{0}}$ & & 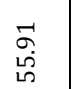 & $\begin{array}{l}\text { นે } \\
\stackrel{0}{0}\end{array}$ & $\overrightarrow{\stackrel{a ̈}{0}}$ \\
\hline & 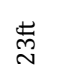 & $\begin{array}{l}\vec{N} \\
\text { ì }\end{array}$ & $\tilde{\tilde{O}}$ & $\stackrel{m}{0}$ & ঙ્ & ָ̃ & : & $\stackrel{0}{\circ}$ & $\underset{+}{\stackrel{\leftrightarrow}{+}}$ & $\stackrel{m}{\leftrightarrow}$ & & $\begin{array}{l}\vec{\sigma} \\
\text { in }\end{array}$ & $\stackrel{\leftrightarrow}{\stackrel{\leftrightarrow}{O}}$ & $\begin{array}{l}\overrightarrow{0} \\
\stackrel{0}{0}\end{array}$ \\
\hline
\end{tabular}




\begin{tabular}{|c|c|c|c|c|c|c|c|c|c|c|c|c|c|c|}
\hline 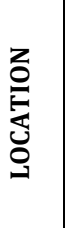 & 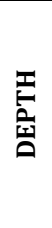 & T & $\dot{8}^{\circ}$ & $\grave{z}$ & 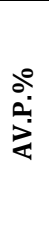 & $\tilde{z}$ & 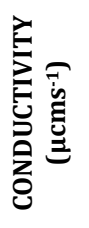 & $\asymp$ & & $\sum^{\infty}$ & 总 & 氙 & $\stackrel{ \pm}{\Sigma}$ & $\stackrel{\infty}{2}$ \\
\hline \multirow{3}{*}{ 柆 } & $\stackrel{ \pm}{m}$ & $\stackrel{m}{\vec{n}}$ & $\stackrel{\mathscr{L}}{\circ}$ & 号 & 号 & $\overrightarrow{\tilde{m}}$ & 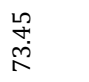 & $\stackrel{\text { L }}{0}$ & $\stackrel{\mathscr{n}}{\stackrel{\sim}{*}}$ & $\vec{g}$ & $\begin{array}{l}\infty \\
\stackrel{\infty}{\circ} \\
\stackrel{\sim}{*}\end{array}$ & $\begin{array}{l}\vec{\nabla} \\
\text { ö }\end{array}$ & $\stackrel{+}{\stackrel{\sim}{\sim}}$ & $\stackrel{\text { N }}{\text { N }}$ \\
\hline & 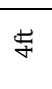 & $\overrightarrow{\text { iे }}$ & $\stackrel{\leftrightarrow}{\circ}$ & $\begin{array}{l}\stackrel{0}{\circ} \\
\stackrel{0}{0}\end{array}$ & 守 & ণ্ণি & $\begin{array}{l}\text { मे } \\
\text { oे }\end{array}$ & $\begin{array}{l}\infty \\
\stackrel{\infty}{0} \\
0\end{array}$ & $\begin{array}{l}\stackrel{0}{1} \\
\text { m }\end{array}$ & 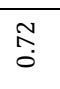 & $\begin{array}{l}\text { No } \\
\infty \\
\infty\end{array}$ & 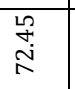 & 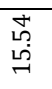 & $\begin{array}{l}\stackrel{\infty}{\sim} \\
\stackrel{0}{0}\end{array}$ \\
\hline & ڤ̆ & $\begin{array}{l}0 \\
\stackrel{\vec{H}}{ }\end{array}$ & $\stackrel{\hat{o}}{0}$ & 苂 & \&. & ָ̃̃ & 离 & $\hat{o}$ & 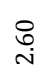 & $\stackrel{\substack{m \\
\sigma}}{\sigma}$ & ્ָخ & $\begin{array}{l}\text { †े } \\
\infty \\
\infty\end{array}$ & $\begin{array}{l}\stackrel{\leftrightarrow}{0} \\
\stackrel{\sim}{\sim}\end{array}$ & $\vec{m}$ \\
\hline$\stackrel{m}{2}$ & $\stackrel{\vec{\sigma}}{\stackrel{\Delta}{\sigma}}$ & $\begin{array}{l}\stackrel{L}{P} \\
\stackrel{5}{\circ}\end{array}$ & $\stackrel{\circ}{\circ}$ & $\begin{array}{l}\tilde{O} \\
\stackrel{0}{0}\end{array}$ & $\stackrel{m}{\stackrel{0}{0}}$ & $\stackrel{\widetilde{N}}{\stackrel{0}{0}}$ & $\stackrel{m}{\stackrel{-}{-}}$ & 吕 & $\underset{+}{\stackrel{\leftrightarrow}{r}}$ & $\stackrel{+}{m}$ & & 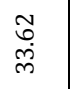 & $\begin{array}{l}\stackrel{\infty}{\infty} \\
\underset{-}{-}\end{array}$ & 앙 \\
\hline \multirow{4}{*}{ 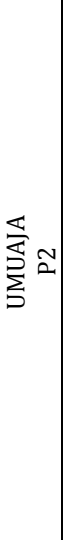 } & 芒 & जे & 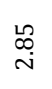 & oे & $\stackrel{\text { ঙ̆ }}{\rightarrow}$ & 范 & $\begin{array}{l}\stackrel{n}{\circ} \\
\stackrel{8}{\circ}\end{array}$ & 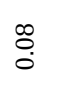 & స్ & $\stackrel{\sim}{\stackrel{\sim}{-}}$ & $\underset{\tilde{N}}{\stackrel{N}{J}}$ & 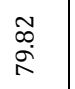 & 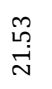 & รั \\
\hline & E & ث̇ & $\underset{-}{\stackrel{\leftrightarrow}{-}}$ & $\stackrel{\text { L }}{0}$ & $\begin{array}{l}\stackrel{R}{0} \\
\stackrel{0}{0}\end{array}$ & $\begin{array}{l}F \\
0\end{array}$ & $\begin{array}{l}\stackrel{0}{0} \\
\text { ¿े. }\end{array}$ & $\begin{array}{l}\tilde{O} \\
\stackrel{0}{0}\end{array}$ & $\overrightarrow{\vec{m}}$ & 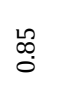 & 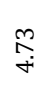 & $\underset{\substack{\infty \\
\infty}}{\vec{\infty}}$ & 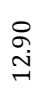 & 앙 \\
\hline & $\underset{\sim}{\stackrel{\rightleftarrows}{\rightleftarrows}}$ & $\underset{\text { in }}{\stackrel{J}{*}}$ & นุ? & $\begin{array}{l}\text { ô } \\
\stackrel{0}{0}\end{array}$ & చี & $\stackrel{\text { N}}{0}$ & $\begin{array}{l}8 \\
\text { : } \\
\text { in }\end{array}$ & $\stackrel{m}{0}$ & $\stackrel{\leftrightarrow}{\stackrel{\leftrightarrow}{-}}$ & 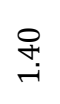 & $\stackrel{8}{\circ}$ & $\begin{array}{l}\text { L } \\
0 \\
0\end{array}$ & $\begin{array}{l}\text { o } \\
\stackrel{0}{0} \\
0\end{array}$ & $\begin{array}{l}0 \\
\stackrel{0}{0}\end{array}$ \\
\hline & $\stackrel{\rightleftarrows}{\stackrel{D}{\circ}}$ & 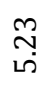 & $\begin{array}{l}\infty \\
\stackrel{\infty}{L} \\
0\end{array}$ & $\begin{array}{l}\text { Oै } \\
\text { Oे }\end{array}$ & $\begin{array}{l}\text { ำ } \\
\text { o }\end{array}$ & & $\begin{array}{l}\stackrel{2}{2} \\
\stackrel{\infty}{\infty}\end{array}$ & $\begin{array}{l}m \\
0 \\
0\end{array}$ & $\begin{array}{l}\text { Oै } \\
\text { ì }\end{array}$ & హ゙ & $\stackrel{\circ}{\stackrel{\circ}{m}}$ & $\begin{array}{l}\text { fे } \\
\text { ने }\end{array}$ & $\begin{array}{l}0 \\
\infty \\
0 \\
0 \\
-1\end{array}$ & $\stackrel{m}{0}$ \\
\hline \multirow{2}{*}{$\approx$} & $\underset{\sim}{\stackrel{\varpi}{\sigma}}$ & $\begin{array}{l}\hat{O} \\
\text { in }\end{array}$ & 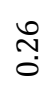 & $\stackrel{\sigma}{\circ}$ & 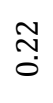 & ন্ & $\begin{array}{l}\text { 노 } \\
\text { 솟 }\end{array}$ & $\begin{array}{l}\tilde{O} \\
\stackrel{0}{0}\end{array}$ & $\begin{array}{l}0 \\
\stackrel{\sim}{v}\end{array}$ & $\begin{array}{l}\text { Lึ } \\
\text { นุ }\end{array}$ & $\underset{\sim}{\stackrel{N}{S}}$ & $\begin{array}{l}\text { ปn } \\
\stackrel{0}{\infty}\end{array}$ & 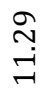 & స̃ \\
\hline & & $\stackrel{\text { N̦ }}{\text { Li }}$ & 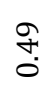 & $\begin{array}{l}\text { oे } \\
\text { o }\end{array}$ & กี & & 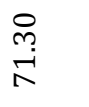 & 웅 & $\begin{array}{l}\text { f } \\
\text { เे }\end{array}$ & 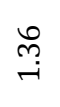 & $\underset{\stackrel{\vec{I}}{ت}}{\vec{ت}}$ & $\begin{array}{l}\text { L } \\
\text { tீ }\end{array}$ & 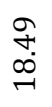 & 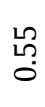 \\
\hline \multirow{2}{*}{$\Xi$} & $\underset{\varpi}{ }$ & $\begin{array}{l}\stackrel{\bigcirc}{\infty} \\
\stackrel{+}{*}\end{array}$ & $\stackrel{\text { N̦}}{0}$ & $\stackrel{m}{0}$ & $\stackrel{\text { ָ̦ }}{\text { Oे }}$ & & $\begin{array}{l}\hat{o} \\
\text { o } \\
\text { in }\end{array}$ & $\begin{array}{l}0 \\
0 \\
0\end{array}$ & $\begin{array}{l}0 \\
m \\
m\end{array}$ & 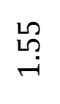 & $\stackrel{\overbrace{}}{\overrightarrow{6}}$ & $\begin{array}{l}\text { H. } \\
\stackrel{0}{\infty}\end{array}$ & $\begin{array}{l}\text { N̦ } \\
\text { Hે }\end{array}$ & 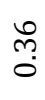 \\
\hline & $\underset{\sim}{\stackrel{\Xi}{ \pm}}$ & $\begin{array}{l}\stackrel{\text { m}}{m} \\
\text { Li }\end{array}$ & $\stackrel{\overbrace{}}{\stackrel{0}{0}}$ & $\begin{array}{l}\tilde{0} \\
0\end{array}$ & $\stackrel{\text { m. }}{\circ}$ & $\begin{array}{l}\stackrel{L}{N} \\
\text { O }\end{array}$ & $\begin{array}{l}\stackrel{0}{1} \\
\dot{m}\end{array}$ & $\begin{array}{l}\text { O̊ } \\
0 \\
0\end{array}$ & $\stackrel{\infty}{\stackrel{\infty}{\rightarrow}}$ & $\underset{N}{\stackrel{n}{0}}$ & $\stackrel{ }{\stackrel{N}{i n}}$ & $\begin{array}{l}\text { I } \\
\text { में }\end{array}$ & $\begin{array}{l}\stackrel{8}{8} \\
\text { i }\end{array}$ & f \\
\hline \multirow{3}{*}{ 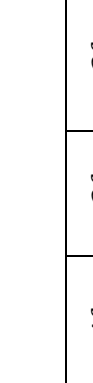 } & $\stackrel{\Xi}{N}$ & 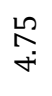 & $\underset{\stackrel{H}{i}}{\stackrel{H}{\prime}}$ & $\begin{array}{l}\infty \\
\stackrel{0}{0} \\
0\end{array}$ & 으. & $\underset{\widetilde{N}}{\tilde{O}}$ & $\begin{array}{l}\text { m. } \\
\text { bे }\end{array}$ & $\stackrel{?}{\stackrel{1}{0}}$ & 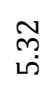 & $\stackrel{\stackrel{\leftrightarrow}{+}}{\leftarrow}$ & $\begin{array}{l}\text { t } \\
\sigma\end{array}$ & $\begin{array}{l}\text { J } \\
\text { J }\end{array}$ & $\underset{7}{\stackrel{7}{ت}}$ & \&. \\
\hline & $\stackrel{ \pm}{m}$ & $\vec{m}$ & 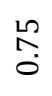 & $\begin{array}{l}\mathcal{N} \\
\dot{0}\end{array}$ & $\begin{array}{l}\text { Lo } \\
\text { L } \\
0\end{array}$ & m̊. & 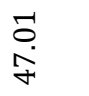 & $\begin{array}{l}\dot{0} \\
0 \\
0\end{array}$ & $\begin{array}{l}\infty \\
\infty \\
+ \\
+\end{array}$ & $\begin{array}{l}\tilde{\sigma} \\
\stackrel{0}{\circ}\end{array}$ & 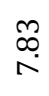 & $\begin{array}{l}\text { ڤొ } \\
\stackrel{0}{\infty}\end{array}$ & $\begin{array}{l}\stackrel{9}{+} \\
\stackrel{1}{-1}\end{array}$ & $\stackrel{\text { f }}{\circ}$ \\
\hline & 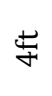 & $\begin{array}{l}\stackrel{m}{+} \\
\text { ம் }\end{array}$ & $\underset{\leftarrow}{*}$ & $\begin{array}{l}\text { Oे } \\
0\end{array}$ & $\stackrel{8}{\circ}$ & m̊ & 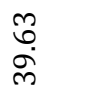 & $\begin{array}{l}\text { L } \\
0\end{array}$ & 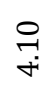 & $\begin{array}{l}\text { Lூ } \\
0\end{array}$ & $\stackrel{ }{ }$ & $\begin{array}{l}\stackrel{+}{~} \\
\text { ă }\end{array}$ & $\begin{array}{l}\stackrel{8}{\circ} \\
\stackrel{-}{-}\end{array}$ & $\begin{array}{l}\stackrel{L}{\circ} \\
\stackrel{0}{0}\end{array}$ \\
\hline
\end{tabular}

Table 3: Chemical Analysis of Subsurface Soil Samples (PPM) 


\begin{tabular}{|c|c|c|c|c|c|}
\hline LOCATION & MEANX & STDEV & SKEWNESS & KIRTOSIS & INTERPRETATION \\
\hline ABK & 1.37 & 0.78 & 0.20 & 2.39 & $\begin{array}{c}\text { Medium grained, moderately sorted, } \\
\text { fine skewed, very leptokurtic sand. }\end{array}$ \\
\hline 0B & 1.0 & 0.97 & 0.25 & 0.79 & $\begin{array}{c}\text { Medium grained, moderately skewed, } \\
\text { Sorted, fine platykurtic sand }\end{array}$ \\
\hline 0B S2 & 1.2 & 0.37 & 0.32 & 0.32 & $\begin{array}{c}\text { Medium grained, moderately skewed, } \\
\text { sorted, fine } \\
\text { platykurtic sand }\end{array}$ \\
\hline ABAVO PI & 1.08 & 1.83 & 0.16 & 1.20 & $\begin{array}{c}\text { Medium grained, poorly sorted, fine } \\
\text { skewed, platykurtic sand }\end{array}$ \\
\hline
\end{tabular}

Table 4: Some Soil

\section{References}

i. Adepeju i. A; Adebeyo A. A; Aduayi E. A; Alofa C. 0. (1979): A Preliminary survey of fertility status of soil in Ondo State under Traditional Cultivation. Ife Journal of Agriculture, Vol. (2) pp 134-149.

ii. Agboola A. A. and Corey E.B. (1976): Nutrient Deficiency survey of Maize in Western Nigeria journal of Science, Vol. 10 pp 1-18.

iii. Barry R.H. and Kurtz L. T. (1945): determination of Total organic and available forms of phosphorus in soil. Journal of Soil Science. Vol. 21, pp. 61-65.

iv. Bouyoucos, G. J. (1962): Hydrometer method improved for marking particle size analysis of soil science. Geological Society of American. Vol. 26. pp. 464-465.

v. Federal Republic of Nigeria Census (199): Nigerian Population Commission publication Lagos.

vi. Ikomi R. B. (2000): The Physico-chemical Hydrology of River Ethiope. Bulletin of Science Association of Nigeria, Vol. 20, pp. 176-188.

vii. Kaizer A. N. and Adaikpoh E. O. (2C06): Some geotechnical properties of soils from Umutu and environ, Southwest Nigeria. Journal of Applied Science and Environmental Management, Vol. 10 (4), pp 41-45.

viii. Kaizer A. N. (2007): Streambed sediment quality from some watersheds in Delta state, Nigeria Journal of Environmental Studies, vol. 3, No pp 33-38.

ix. $\quad$ Kaizer A. N. (2007): Trace metal and Hydrocarbon Composition of soils in Umutu and environs, Southwestern Nigeria Journal of Science and Environment, Vol. 6, pp 140-146.

x. Kent A. A. (1998): Nitrogen in Soils. Http:/www.mit.edu.overture.com. Browsed 10 ${ }^{\text {th }}$ February, 2009.

xi. Kent A. A. (2001): Phosphorus Retention by soil in Minnesota River Http:/www.mit.edu.overture.com. Browsed 10th February, 2009.

xii. Schollenberger D. J. and Simons R. H. (1945): Determination of Exchange Capacity and Exchangeable Bases in Soils, Ammonium Acetate Method. Journal of Soil Science, VOI. 51, pp. 761-779.

xiii. Walkley A. and Black A. I. (1934): An Examination of the Degtarett modification of the chronic and Titration method. Journal of Soil Science, Vol. 37, pp 29-38. 